\title{
Soft semiseparated sets via operations
}

\author{
B. Jaya Bharathi ${ }^{1 *}$ and P. Gomathi sundari ${ }^{2}$
}

\begin{abstract}
In this paper, we introduce the notion $\gamma$-soft semiseparated sets and study some of their basic properties.

Keywords

Soft topological spaces, $\gamma$-soft semiseparated set, $\gamma$-soft semiconnected space.

AMS Subject Classification 54D05, 54C08, 54B05.

${ }_{1,2}$ Department of Mathematics, Rajah Serfoji Government College, Affiliated to Bharathidasan University, Thanjavur, Tamil Nadu-613005, India. ${ }^{\star}$ Corresponding author: ${ }^{1}$ vbhavatharani2011@gmail.com; ${ }^{2}$ rsgcgomathi18@gmail.com

Article History: Received 13 August 2019; Accepted 22 November 2019

(C)2020 MJM
\end{abstract}

\section{Contents}

1 Introduction. 42

2

Preliminaries $. \ldots \ldots \ldots \ldots \ldots \ldots \ldots \ldots \ldots \ldots \ldots \ldots, 42$

On operation-soft semiseparated sets ..........43

Operation-soft semiconnected spaces...........44

References ............................. 46

\section{Introduction}

The concept of soft sets was first introduced by Molodtsov [11]. After the introduction of the definition of a soft sets by Molodtsov, a large number of topologists have turned their attention to the generalization of different concepts of a classical sets in this sets. Recently, the concept of soft topological spaces was introduced and studied by Shabir and Naz [17]. A Good number of results are studied in this paper. The study of topological properties via operations was introduced and studied by Biswas and Prasannan in [2]. In this paper, we introduce the notion $\gamma$-soft semiseparated sets and study some of their basic properties. Also the concept of $\gamma$-soft semiconnected spaces are also introduced and studied in this paper.

\section{Preliminaries}

Let $U$ be an initial universe set and $E_{U}$ be a collection of all possible parameters with respect to $U$, where parameters are the characteristics or properties of objects in $U$. We will call $E_{U}$ the universe set of parameters with respect to $U$.

Definition 2.1. [11] A pair $(F, A)$ is called a soft set over $U$ if $A \subset E_{U}$ and $F: A \rightarrow P(U)$, where $P(U)$ is the set of all subsets of $U$.

Definition 2.2. [6] Let $U$ be an initial universe set and $E_{U}$ be a universe set of parameters. Let $(F, A)$ and $(G, B)$ be soft sets over a common universe set $U$ and $A, B \subset E$. Then $(F, A)$ is a subset of $(G, B)$, denoted by $(F, A) \widetilde{\subset}(G, B)$, if $A \subset B$ and for all e $\in A, F(e) \subset G(e)$. Also $(F, A)$ equals $(G, B)$, denoted by $(F, A)=(G, B)$, if $(F, A) \widetilde{\subset}(G, B)$ and $(G, B) \widetilde{\subset}(F, A)$.

Definition 2.3. [12] A soft set $(F, A)$ over $U$ is called a null soft set, denoted by $\widetilde{\emptyset}$, if $e \in A, F(e)=\emptyset$.

Definition 2.4. [12] A soft set $(F, A)$ over $U$ is called an absolute soft set, denoted by $\widetilde{A}$, if $e \in A, F(e)=U$.

Definition 2.5. [12] The union of two soft sets $(F, A)$ and $(G, B)$ over a common universe $U$ is the soft set $(H, C)$, where $C=A \cup B$, and for all $e \in C$,

$$
H(e)= \begin{cases}F(e) & \text { if } e \in A \backslash B, \\ G(e) & \text { if } e \in B \backslash A, \\ F(e) \cup G(e) & \text { if } e \in B \cap A .\end{cases}
$$

We write $(F, A) \cup(G, B)=(H, C)$.

Definition 2.6. [6] The intersection of two soft sets of $(F, A)$ and $(G, B)$ over a common universe $U$ is the soft set $(H, C)$, where $C=A \cap B$, and for all $e \in C, H(e)=F(e) \cap G(e)$. We write $(F, A) \cap(G, B)=(H, C)$.

Now we recall some definitions and results defined and discussed in [16, 17]. Henceforth, let $X$ be an initial universe set and $E$ be the fixed nonempty set of parameter with respect to $X$ unless otherwise specified. 
Definition 2.7. For a soft set $(F, A)$ over $U$, the relative complement of $(F, A)$ is denoted by $(F, A)^{\prime}$ and is defined by $(F, A)^{\prime}=\left(F^{\prime}, A\right)$, where $F^{\prime}: A \rightarrow P(U)$ is a mapping given by $F^{\prime}(e)=U \backslash F(e)$ for all $e \in A$.

Definition 2.8. Let $\tau$ be the collection of soft sets over $X$, then $\tau$ is called a soft topology on $X$ if $\tau$ satisfies the following axioms.

1. $\widetilde{\emptyset}, \widetilde{X}$ belong to $\tau$.

2. The union of any number of soft sets in $\tau$ belongs to $\tau$.

3. The intersection of any two soft sets in $\tau$ belongs to $\tau$.

The triplet $(X, \tau, E)$ is called a soft topological space.

Definition 2.9. Let $(X, \tau, E)$ be a soft topological space, then the members of $\tau$ are said to be soft open sets in $X$.

Definition 2.10. Let $(X, \tau, E)$ be a soft topological space. A soft set $(F, E)$ over $X$ is said to be a soft closed set in $X$, if its relative complement $(F, E)^{\prime}$ belongs to $\tau$.

Proposition 2.11. Let $(X, \tau, E)$ be a soft topological space. Then one has the following

1. $\widetilde{\emptyset}, \widetilde{X}$ are soft closed sets over $X$.

2. The intersection of any number of soft closed sets is a soft closed set over $X$.

3. The union of any two soft closed sets is a soft closed set over $X$.

Definition 2.12. Let $(X, \tau, E)$ be a soft topological space and $(A, E)$ a soft set over $X$.

1. The soft interior of $(A, E)$ is the soft set $\operatorname{Int}(A, E)=$ $\cup\{(O, E):(O, E)$ is soft open and $(O, E) \widetilde{C}(A, E)\}$.

2. The soft closure of $(A, E)$ is the soft set $\mathrm{Cl}(A, E)=$ $\cap\{(F, E):(F, E)$ is soft closed and $(A, E) \widetilde{C}(F, E)\}$.

Definition 2.13. Let $(F, E)$ be a soft set over $X$ and $x \in X$. We say that $x \in(F, E)$ read as $x$ belongs to the soft set $(F, E)$, whenever $x \in F(\alpha)$ for all $\alpha \in E$. Note that for $x \in X, x \notin$ $(F, E)$ if $x \notin F(\alpha)$ for $\alpha \in E$.

Definition 2.14. Let $x \in X$, then $(x, E)$ denotes the soft set over $X$ for which $x(\alpha)=\{x\}$ for all $\alpha \in E$.

Definition 2.15. [2] An operation on a soft topology $\tau$ over $X$ is called a $\gamma$-operation if a mapping from $\tau$ to the set $P(X)^{E}$ and defined by $\gamma: \tau \rightarrow P(X)^{E}$ such that for each $(V, E) \in \tau$, $(V, E) \widetilde{\subset} \gamma(V, E)$.

Definition 2.16. [2] A soft set $(P, E)$ is said to be $\gamma$-soft open set if for each $x \tilde{\in}(P, E)$, there exists a soft open set $(V, E)$ such that $x \widetilde{\in}(V, E) \widetilde{\subset} \gamma(V, E) \widetilde{C}(P, E)$. The complement of a $\gamma$-soft open set is called a $\gamma$-soft closed set. The family of all $\gamma$-soft open sets of $(X, \tau, E, \gamma)$ is denoted by $\tau_{\gamma}$.
Definition 2.17. [2] Let $(X, \tau, E, \gamma)$ be an operation-soft topological space and $(A, E)$ a soft set over $X$. Then

1. the $\tau_{\gamma}$-soft interior of $(A, E)$ is the soft set $\tau_{\gamma}-\operatorname{Int}(A, E)=$ $\cup\{(O, E):(O, E)$ is $\gamma$-soft open and $(O, E) \widetilde{C}(A, E)\}$.

2. the $\tau_{\gamma}$-soft closure of $(A, E)$ is the soft set $\tau_{\gamma}-\mathrm{Cl}(A, E)=$ $\cap\{(F, E):(F, E)$ is $\gamma$-soft closed and $(A, E) \widetilde{\subset}(F, E)\}$.

Lemma 2.18. Let $(X, \tau, E, \gamma)$ be an operation-soft topological space. Then

1. for every $\gamma$-soft open set $(G, E)$ and every soft subset $(A, E)$ over $X$ we have $\tau_{\gamma^{-}} \mathrm{Cl}((A, E)) \cap(G, E) \subset \tau_{\gamma^{-}}$ $\mathrm{Cl}((A, E) \cap(G, E))$,

2. for every $\gamma$-soft closed set $(F, E)$ and every soft subset $(A, E)$ over $X$ we have $\tau_{\gamma^{-}} \operatorname{Int}\left((A, E) \cap(F, E) \subset \tau_{\gamma^{-}}\right.$ $\operatorname{Int}((A, E)) \cap(F, E)$.

Definition 2.19. A subset $(A, E)$ of an operation-soft topological space $(X, \tau, E, \gamma)$ is said to be $\gamma$-soft semiopen [7] if $(A, E) \subset \tau_{\gamma}-\mathrm{Cl}\left(\tau_{\gamma}-\operatorname{Int}((A, E))\right)$.

The complement of a $\gamma$-soft semiopen set is called a $\gamma$ soft semiclosed set. The family of all $\gamma$-soft semiopen sets of $(X, \tau, E, \gamma)$ is denoted by $\gamma-\operatorname{SSO}(X)$.

Definition 2.20. [7] Let $(X, \tau, E, \gamma)$ be an operation-soft topological space and $(A, E)$ a soft set over $X$. Then

1. $\tau_{\gamma}$-s $\operatorname{Int}(A, E)=\cup\{(O, E):(O, E)$ is $\gamma$-soft semiopen and $(O, E) \widetilde{C}(A, E)\}$.

2. $\tau_{\gamma^{-s}} \mathrm{Cl}(A, E)=\cap\{(F, E):(F, E)$ is $\gamma$-soft semiclosed and $(A, E) \widetilde{\subset}(F, E)\}$.

\section{On operation-soft semiseparated sets}

Definition 3.1. Two nonempty soft subsets $(A, E)$ and $(B, E)$ of an operation-soft topological space $(X, \tau, E, \gamma)$ are said to be $\gamma$-soft semiseparated if $(A, E) \widetilde{\cap} \tau_{\gamma^{-}} \mathrm{Cl}((B, E))=\tau_{\gamma^{-}}$ ${ }_{s} \mathrm{Cl}((A, E)) \widetilde{\cap}(B, E)=\widetilde{\emptyset}$. If $\widetilde{X}=(A, E) \widetilde{\cup}(B, E)$ such that $(A, E)$ and $(B, E)$ are $\gamma$-soft semiseparated sets, then we say that $(A, E)$ and $(B, E)$ form a $\gamma$-soft semiseparation of $\widetilde{X}$.

Remark 3.2. Each two $\gamma$-soft semiseparated sets are always disjoint, since $(A, E) \widetilde{\cap}(B, E) \widetilde{C}(A, E) \widetilde{\cap} \tau_{\gamma^{-}} \operatorname{Cl}((B, E))=\widetilde{\emptyset}$.

Theorem 3.3. For the soft subsets $(A, E)$ and $(B, E)$ of an operation-soft topological space $(X, \tau, E, \gamma)$, the following statements are equivalent:

1. $(A, E)$ and $(B, E)$ are $\gamma$-soft semiseparated.

2. There exist $\gamma$-soft semiclosed sets $\left(F_{1}, E\right),\left(F_{2}, E\right)$ satisfying $(A, E) \widetilde{C}\left(F_{1}, E\right) \widetilde{\subset} \widetilde{X} \backslash(B, E)$ and $(B, E) \widetilde{C}\left(F_{2}, E\right)$ $\tilde{\subset} \widetilde{X} \backslash(A, E)$. 
3. There exist $\gamma$-soft semiopen sets $\left(G_{1}, E\right)$ and $\left(G_{2}, E\right)$ satisfying $(A, E) \widetilde{C}\left(G_{1}, E\right)$ $\widetilde{\subset}(\widetilde{X} \backslash(B, E))$ and $(B, E) \widetilde{C}\left(G_{2}, E\right) \widetilde{\subset} \widetilde{X} \backslash(A, E)$.

Proof. The proof is clear.

Proposition 3.4. Let $(A, E)$ and $(B, E)$ be soft subsets of an operation-soft topological space $(X, \tau, E, \gamma)$. If $(A, E)$ and $(B, E)$ are $\gamma$-soft semiseparated, $\widetilde{\emptyset} \neq(C, E) \widetilde{C}(A, E)$ and $\widetilde{\emptyset} \neq(D, E) \widetilde{C}(B, E)$, then $(C, E)$ and $(D, E)$ are $\gamma$-soft semiseparated.

Proof. Since $(A, E)$ and $(B, E)$ are $\gamma$-soft semiseparated sets, $(A, E) \widetilde{\cap} \tau_{\gamma^{-}} \operatorname{Cl}((B, E))=\widetilde{\emptyset}$ and $\tau_{\gamma^{-}} \operatorname{Cl}((A, E)) \widetilde{\cap}(B, E)=\widetilde{\emptyset}$. Then $(C, E) \widetilde{C}(A, E), \tau_{\gamma^{-} s} \operatorname{Cl}((C, E)) \widetilde{\cap}(D, E)=\widetilde{\emptyset}$. Similarly, we have $(C, E) \widetilde{\cap} \tau_{\gamma^{-}} \operatorname{Cl}((D, E))=\widetilde{\emptyset}$. Therefore, $(C, E)$ and $(D, E)$ are $\gamma$-soft semiseparated sets.

Theorem 3.5. If $(A, E)$ and $(B, E)$ are $\gamma$-soft semiseparated sets and $(S, E)$ is a $\gamma$-soft semiclosed subset of an operationsoft topological space such that $(S, E)=(A, E) \widetilde{\cup}(B, E)$, then $(A, E)$ and $(B, E)$ are $\gamma$-soft semiclosed sets.

Proof. Let $(S, E)=(A, E) \widetilde{\cup}(B, E)$, where $\tau_{\gamma-s} \operatorname{Cl}((A, E)) \widetilde{\cap}$ $(B, E)=\widetilde{\emptyset}=(A, E) \widetilde{\cap} \tau_{\gamma^{-}} \mathrm{Cl}((B, E))$. It is clear that $(S, E) \widetilde{\cap} \tau_{\gamma^{-}}$ $s \mathrm{Cl}((A, E))=((A, E) \widetilde{\cup}(B, E)) \widetilde{\cap} \tau_{\gamma-s} \mathrm{Cl}((A, E))=(A, E)$. As the intersection of $\gamma$-soft semiclosed sets is $\gamma$-soft semiclosed, $(A, E)$ is $\gamma$-soft semiclosed. Similarly $(B, E)$ is $\gamma$-soft semiclosed.

Theorem 3.6. Let $(A, E)$ and $(B, E)$ be nonempty soft subsets in an operation-soft topological space $(X, \tau, E, \gamma)$. The following statements hold:

1. If $(A, E)$ and $(B, E)$ are $\gamma$-soft semiseparated such that $\left(A_{1}, E\right) \widetilde{C}(A, E),\left(B_{1}, E\right) \widetilde{C}(B, E)$, then $\left(A_{1}, E\right),\left(B_{1}, E\right)$ are so.

2. If $(A, E) \widetilde{\cap}(B, E)=\widetilde{\emptyset}$ such that $(A, E)$ and $(B, E)$ are $\gamma$-soft semiclosed ( $\gamma$-soft semiopen), then $(A, E)$ and $(B, E)$ are $\gamma$-soft semiseparated.

3. If $(A, E),(B, E)$ are $\gamma$-soft semiclosed ( $\gamma$-soft semiopen) and $(H, E)=(A, E) \cap(\widetilde{X} \backslash(B, E))$ and $(G, E)=(B, E) \cap$ $(\widetilde{X} \backslash(A, E))$, then $(H, E)$ and $(G, E)$ are $\gamma$-soft semiseparated.

Proof. (1). Since $\left(A_{1}, E\right) \widetilde{C}(A, E)$, we have $\tau_{\gamma^{-} s} \operatorname{Cl}\left(\left(A_{1}, E\right)\right) \widetilde{C}$ $\tau_{\gamma^{-}} \mathrm{Cl}((A, E))$. Then $(B, E) \widetilde{\cap} \tau_{\gamma^{-s}} \mathrm{Cl}((A, E))=\widetilde{\emptyset}$ implies that $\left(B_{1}, E\right) \widetilde{\cap} \tau_{\gamma^{-} s} \operatorname{Cl}((A, E))=\widetilde{\emptyset}$ and $\left(B_{1}, E\right) \widetilde{\cap} \tau_{\gamma^{-}} \operatorname{Cl}\left(\left(A_{1}, E\right)\right)=$ $\widetilde{\emptyset}$. Similarly $\left(A_{1}, E\right) \widetilde{\cap} \tau_{\gamma^{-} s} \operatorname{Cl}\left(\left(B_{1}, E\right)\right)=\widetilde{\emptyset}$. Hence $\left(A_{1}, E\right)$ and $\left(B_{1}, E\right)$ are $\gamma$-soft semiseparated.

(2). Since $(A, E)=\tau_{\gamma^{-}} \mathrm{Cl}((A, E)),(B, E)=\tau_{\gamma^{-}} \mathrm{Cl}((B, E))$ and $(A, E) \widetilde{\cap}(B, E)=\widetilde{\emptyset}, \tau_{\gamma^{-} s} \mathrm{Cl}((A, E)) \widetilde{\cap}(B, E)=\widetilde{\emptyset}$ and $\tau_{\gamma^{-}}$ $s \mathrm{Cl}((B, E)) \widetilde{\cap}(A, E)=\widetilde{\emptyset}$. Hence $(A, E)$ and $(B, E)$ are $\gamma$-soft semiseparated sets. If $(A, E)$ and $(B, E)$ are $\gamma$-soft semiopen, then their complements are $\gamma$-soft semiclosed.
(3). If $(A, E)$ and $(B, E)$ are $\gamma$-soft semiopen, then $\tilde{X} \backslash(A, E)$ and $\widetilde{X} \backslash(B, E)$ are $\gamma$-soft semiclosed. Since $(H, E) \widetilde{\subset} \widetilde{X} \backslash(B, E)$, $\tau_{\gamma^{-}} \operatorname{Cl}((H, E)) \widetilde{C} \tau_{\gamma^{-} s} \operatorname{Cl}(\widetilde{X} \backslash(B, E))=\widetilde{X} \backslash(B, E)$ and hence $\tau_{\gamma^{-}}$ $s \mathrm{Cl}((H, E)) \widetilde{\cap}(B, E)=\widetilde{\emptyset}$. Thus $(G, E) \widetilde{\cap} \tau_{\gamma^{-}} \operatorname{Cl}((H, E))=\widetilde{\emptyset}$. Similarly, $(H, E) \widetilde{\cap} \tau_{\gamma^{-}} \operatorname{Cl}((G, E))=\widetilde{\emptyset}$. Hence $(H, E)$ and $(G, E)$ are $\gamma$-soft semiseparated sets.

Theorem 3.7. The soft sets $(A, E)$ and $(B, E)$ of an operationsoft topological space are $\gamma$-soft semiseparated if, and only if there exist $(U, E),(V, E) \in \gamma S S O(X)$ such that $(A, E) \widetilde{\subset}(U, E)$, $(B, E) \widetilde{C}(V, E),(A, E) \widetilde{\cap}(V, E)=\widetilde{\emptyset}$ and $(B, E) \widetilde{\cap}(U, E)=\widetilde{\emptyset}$.

Proof. Suppose that $(V, E)=\widetilde{X} \backslash \tau_{\gamma^{-} s} \mathrm{Cl}((A, E))$ and $(U, E)=$ $\widetilde{X} \backslash \tau_{\gamma^{-}} \operatorname{Cl}((B, E))$. Then $(U, E),(V, E) \in \gamma S S O(X)$ such that $(A, E) \widetilde{C}(U, E),(B, E) \widetilde{\subset}(V, E),(A, E) \widetilde{\cap}(V, E)=\widetilde{\emptyset}$ and $(B, E)$ $\widetilde{\cap}(V, E)=\widetilde{\emptyset}$. On the other hand, let $(U, E),(V, E) \in \gamma \operatorname{SSO}(X)$ such that $(A, E) \widetilde{\subset}(U, E),(B, E) \widetilde{\subset}(V, E),(A, E) \widetilde{\cap}(V, E)=\widetilde{\emptyset}$ and $(B, E) \widetilde{\cap}(U, E)=\widetilde{\emptyset}$. Since $\widetilde{X} \backslash(V, E)$ and $\widetilde{X} \backslash(U, E)$ are $\gamma$-soft semiclosed sets, $\tau_{\gamma^{-}} \operatorname{Cl}((A, E)) \widetilde{\subset} \widetilde{X} \backslash(V, E) \widetilde{\subset} \widetilde{X} \backslash(B, E)$ and $\tau_{\gamma^{-} s} \operatorname{Cl}((B, E)) \widetilde{\subset} \widetilde{X} \backslash(U, E) \widetilde{C} \widetilde{X} \backslash(A, E)$. It follows that $\tau_{\gamma^{-}}$ $s \mathrm{Cl}((A, E)) \widetilde{\cap}(B, E)=\widetilde{\emptyset}$ and $\tau_{\gamma^{-}} s \mathrm{Cl}((B, E)) \widetilde{\cap}(A, E)=\widetilde{\emptyset}$.

Theorem 3.8. Let $(A, E)$ and $(B, E)$ be nonempty disjoint soft subsets of an operation-soft topological space $(X, \tau, E, \gamma)$ and $(G, E)=(A, E) \widetilde{\cup}(B, E)$. Then $(A, E)$ and $(B, E)$ are $\gamma$-soft semiseparated if and only if each of $(A, E)$ and $(B, E)$ are $\gamma$-soft semiclosed ( $\gamma$-soft semiopen) in $(G, E)$.

Proof. Let $(A, E)$ and $(B, E)$ are $\gamma$-soft semiseparated sets. By Definition 3.1, $(A, E)$ contains no $\gamma$-soft semilimit points of $(B, E)$. Then $(B, E)$ contains all $\gamma$-soft semilimit points of $(B, E)$ which are in $(A, E) \widetilde{\cup}(B, E)$ and $(B, E)$ is $\gamma$-soft semiclosed in $(A, E) \widetilde{\cup}(B, E)$. Therefore $(B, E)$ is $\gamma$-soft semiclosed in $(G, E)$. Similarly $(A, E)$ is $\gamma$-soft semiclosed in $(G, E)$.

Theorem 3.9. Let $(X, \tau, E, \gamma)$ be an operation-soft topological space. If $(A, E)$ and $(B, E)$ are $\gamma$-soft semiseparation of $\widetilde{X}$ itself, then $(A, E)$ and $(B, E)$ are $\gamma$-soft semiclosed sets of $(X, \tau, E, \gamma)$.

Proof. Since $(A, E)$ and $(B, E)$ are $\gamma$-soft semiseparated, we have $(A, E) \widetilde{\cap} \tau_{\gamma^{-}} \operatorname{Cl}((B, E))=\tau_{\gamma^{-}} \operatorname{Cl}((A, E)) \widetilde{\cap}(B, E)=\widetilde{\emptyset}$. Then $(A, E) \widetilde{\cap} \tau_{\gamma-s} \operatorname{Cl}((B, E))=\widetilde{\emptyset}$ if, and only if $(B, E)$ is $\gamma$-soft semiclosed in $(A, E) \widetilde{\cup}(B, E)=\widetilde{X}$. Similarly, we can show that $(A, E)$ is $\gamma$-soft semiclosed in $\widetilde{X}$.

\section{Operation-soft semiconnected spaces}

Definition 4.1. A subset $(A, E)$ of an operation-soft topological space $(X, \tau, E, \gamma)$ is said to be $\gamma$-soft semiconnected if it cannot be expressed as the union of two $\gamma$-soft semiseparated sets. Otherwise, the set $(A, E)$ is called $\gamma$-soft semidisconnected. 
Lemma 4.2. Let $(A, E) \widetilde{C}(B, E) \widetilde{\cup}(C, E)$ such that $(A, E)$ be a nonempty $\gamma$-soft semiconnected set in an operation-soft topological space $(X, \tau, E, \gamma)$ and $(B, E),(C, E)$ be $\gamma$-soft semiseparated sets. Then only one of the following conditions holds:

$$
\begin{aligned}
& \text { 1. }(A, E) \widetilde{\subset}(B, E) \text { and }(A, E) \widetilde{\cap}(C, E)=\widetilde{\emptyset} . \\
& \text { 2. }(A, E) \widetilde{C}(C, E) \text { and }(A, E) \widetilde{\cap}(B, E)=\widetilde{\emptyset} .
\end{aligned}
$$

Proof. Since $(A, E) \widetilde{\cap}(C, E)=\widetilde{\emptyset}$, we have $(A, E) \widetilde{C}(B, E)$. If $(A, E) \widetilde{\cap}(B, E)=\widetilde{\emptyset}$, then $(A, E) \widetilde{C}(C, E)$. Since $(A, E) \widetilde{\subset}(B, E)$ $\widetilde{\cap}(C, E)$, then both $(A, E) \widetilde{\cap}(B, E)=\widetilde{\emptyset}$ and $(A, E) \widetilde{\cap}(C, E)=\widetilde{\emptyset}$ cannot hold. Similarly, suppose that $(A, E) \widetilde{\cap}(B, E) \neq \widetilde{\emptyset}$ and $(A, E) \widetilde{\cap}(C, E) \neq \widetilde{\emptyset}$, then by Theorem $3.6(1),(A, E) \widetilde{\cap}(B, E)$ and $(A, E) \widetilde{\cap}(C, E)$ are $\gamma$-soft semiseparated sets such that $(A, E)=((A, E) \widetilde{\cap}(B, E)) \widetilde{\cup}((A, E) \widetilde{\cap}(C, E))$ which contradicts with the $\gamma$-soft semiconnectedness of $(A, E)$. Hence one of the conditions (1) and (2) must be hold.

Theorem 4.3. If a $\gamma$-soft semiconnected soft set $(S, E)$ of an operation-soft topological space $(X, \tau, E, \gamma)$ is contained in $(A, E) \widetilde{\cup}(B, E)$, where $(A, E)$ and $(B, E)$ are $\gamma$-soft semiseparated sets, then either $(S, E) \widetilde{C}(A, E)$ or $(S, E) \widetilde{C}(B, E)$.

Proof. Let $(S, E)=((S, E) \widetilde{\cap}(A, E)) \widetilde{\cup}((S, E) \widetilde{\cup}(B, E))$ where $(S, E) \widetilde{\cap}(A, E)$ and $(S, E) \widetilde{\cap}(B, E)$ are $\gamma$-soft semiseparated sets. So either $(S, E) \widetilde{\cap}(A, E)=\widetilde{\emptyset}$ or $(S, E) \widetilde{\cap}(B, E)=\widetilde{\emptyset}$ and hence either $(S, E) \widetilde{C}(B, E)$ or $(S, E) \widetilde{C}(A, E)$.

Theorem 4.4. A soft subset $(M, E)$ of an operation-soft topological space $(X, \tau, E, \gamma)$ is a $\gamma$-soft semiconnected if there exists a $\gamma$-soft semiconnected set $(C, E)$ such that $(C, E) \widetilde{C}(M, E)$ $\widetilde{\subset} \tau_{\gamma-s} \mathrm{Cl}((C, E))$.

Proof. Let $(M, E)=(A, E) \widetilde{\cup}(B, E)$, where $(A, E)$ and $(B, E)$ are $\gamma$-soft semiseparated sets. Then either $(C, E) \widetilde{C}(A, E)$ and $(C, E) \widetilde{C}(B, E)$ and hence either $(M, E) \subset \tau_{\gamma^{-}} \mathrm{Cl}((C, E)) \subset \tau_{\gamma^{-}}$ $s \mathrm{Cl}((A, E)) \widetilde{C}(\widetilde{X} \backslash(B, E))$ or $(M, E) \widetilde{C}(\widetilde{X} \backslash(A, E))$. Therefore either $(B, E)=\widetilde{\emptyset}$ or $(A, E)=\widetilde{\emptyset}$.

Corollary 4.5. If $(C, E)$ is a $\gamma$-soft semiconnected soft set of an operation-soft topological space $(X, \tau, E, \gamma)$, then $\tau_{\gamma^{-}}$ $s \mathrm{Cl}((C, E))$ is so.

Proof. Follows from Theorem 4.4.

Theorem 4.6. If $\left\{\left(M_{\alpha}, E\right): \alpha \in \Delta\right\}$ is a family of $\gamma$-soft semiconnected sets of an operation-soft topological space $(X, \tau, E, \gamma)$ satisfying the property that any two of which are not $\gamma$-soft semiseparated, then $(M, E)=\underset{\alpha \in \Delta}{\cup}\left(M_{\alpha}, E\right)$ is $\gamma$-soft semiconnected.

Proof. Let $(M, E)=(A, E) \widetilde{\cup}(B, E)$, where $(A, E)$ and $(B, E)$ are $\gamma$-soft semiseparated sets. Then for each $\alpha \in \Delta$ either $\left(M_{\alpha}, E\right) \widetilde{\subset}(A, E)$ or $\left(M_{\alpha}, E\right) \widetilde{\subset}(B, E)$. Since any two members of the family $\left\{\left(M_{\alpha}, E\right): \alpha \in \Delta\right\}$ are not $\gamma$-soft semiseparated, either $\left(M_{\alpha}, E\right) \widetilde{C}(A, E)$ for each $\alpha \in \Delta$ or $\left(M_{\alpha}, E\right) \widetilde{C}(B, E)$ for each $\alpha \in \Delta$. So either $(B, E)=\widetilde{\emptyset}$ or $(A, E)=\widetilde{\emptyset}$.
Corollary 4.7. If $(M, E)=\cup_{\alpha \in \Delta}\left(M_{\alpha}, E\right)$, where each $\left(M_{\alpha}, E\right)$ is $\gamma$-soft semiconnected set in an operation-soft topological space $(X, \tau, E, \gamma)$ and also $\left(M_{\alpha}, E\right) \widetilde{\cap}\left(M_{\alpha^{\prime}}, E\right) \neq \widetilde{\emptyset}$ for $\alpha, \alpha^{\prime} \in$ $\Delta$, then $(M, E)$ is $\gamma$-soft semiconnected.

Proof. Follows from Theorem 4.6.

Corollary 4.8. If $(M, E)=\underset{\alpha \in \Delta}{\cup}\left(M_{\alpha}, E\right)$, where each $\left(M_{\alpha}, E\right)$ is $\gamma$-soft semiconnected in an operation-soft topological space $(X, \tau, E, \gamma)$ and $\cap_{\alpha \in \Delta}\left(M_{\alpha}, E\right) \neq \widetilde{\emptyset}$ for each $\alpha \in \Delta$, then $(M, E)$ is $\gamma$-soft semiconnected.

Proof. Suppose that $\cup_{\alpha \in \Delta}\left(M_{\alpha}, E\right)$ is not $\gamma$-soft semiconnected. Then $\underset{\alpha \in \Delta}{\cup}\left(M_{\alpha}, E\right)=(H, E) \widetilde{\cup}(G, E)$, where $(H, E)$ and $(G, E)$ are $\gamma$-soft semiseparated sets in $\widetilde{X}$. Since $\bigcap_{\alpha \in \Delta}\left(M_{\alpha}, E\right) \neq \widetilde{\emptyset}$, we have a soft point $e_{M} \widetilde{\epsilon} \bigcap_{\alpha \in \Delta}\left(M_{\alpha}, E\right)$. Since $e_{M} \in \cup_{\alpha \in \Delta}\left(M_{\alpha}, E\right)$, either $e_{M} \in(G, E)$ or $e_{M} \in(H, E)$. Suppose that $e_{M} \in(H, E)$. Since $e_{M} \in\left(M_{\alpha}, E\right)$ for each $\alpha \in \Delta$, then $\left(M_{\alpha}, E\right)$ and $(H, E)$ intersect for each $\alpha \in \Delta$. By Theorem 4.3, $\left(M_{\alpha}, E\right) \widetilde{\subset}(H, E)$ or $\left(M_{\alpha}, E\right) \widetilde{C}(G, E)$. Since $(H, E)$ and $(G, E)$ are disjoint, $\left(M_{\alpha}, E\right) \widetilde{C}(H, E)$ for all $\alpha \in \Delta$ and hence $\cup_{\alpha \in \Delta}\left(M_{\alpha}, E\right) \widetilde{\subset}(H, E)$. Then $(G, E)=\widetilde{\emptyset}$, which is a contradiction. Suppose that $e_{M} \in(G, E)$. By similar way, we have $(H, E)=\widetilde{\emptyset}$, which is a contradiction. Thus $\cup_{\alpha \in \Delta}\left(M_{\alpha}, E\right)$ is $\gamma$-soft semiconnected.

Theorem 4.9. The following statements are equivalent for an operation-soft topological space $(X, \tau, E, \gamma)$ :

1. $\widetilde{X}$ is $\gamma$-soft semiconnected.

2. $\widetilde{X}$ can not be expressed as the union of two nonempty disjoint $\gamma$-soft semiopen sets.

3. $\widetilde{X}$ contains no nonempty soft subset which is both $\gamma$-soft semiopen and $\gamma$-soft semiclosed.

Proof. (1) $\Rightarrow$ (2): Suppose that $\widetilde{X}$ is $\gamma$-soft semiconnected and if $\widetilde{X}$ can be expressed as the union of two nonempty disjoint sets $(A, E)$ and $(B, E)$ such that $(A, E)$ and $(B, E)$ are $\gamma$-soft semiopen sets. Consequently $(A, E) \widetilde{\subset} \widetilde{X} \backslash(B, E)$. Then $\tau_{\gamma^{-}} \operatorname{Cl}((A, E)) \widetilde{\subset} \tau_{\gamma^{-} s} \mathrm{Cl}(\widetilde{X} \backslash(B, E))=\widetilde{X} \backslash(B, E)$. Therefore, $\tau_{\gamma^{-} s} \mathrm{Cl}((A, E)) \widetilde{\cap}(B, E)=\widetilde{\emptyset}$. Similarly we can prove $(A, E) \widetilde{\cap} \tau_{\gamma^{-}} \mathrm{Cl}((B, E))=\widetilde{\emptyset}$. This is a contradiction to the fact that $X$ is $\gamma$-soft semiconnected. Then $\widetilde{X}$ cannot be expressed as the union of two nonempty disjoint $\gamma$-soft semiopen sets.

$(2) \Rightarrow(3)$ : Suppose that $\widetilde{X}$ cannot be expressed as the union of two nonempty disjoint sets $(A, E)$ and $(B, E)$ such that $(A, E)$ and $(B, E)$ are $\gamma$-soft semiopen sets. If $\widetilde{X}$ contains a nonempty proper subset $(A, E)$ which is both $\gamma$-soft semiopen and $\gamma$-soft semiclosed. Then $\widetilde{X}=(A, E) \widetilde{\cup}(\widetilde{X} \backslash(A, E))$. Hence $(A, E)$ and $\widetilde{X} \backslash(A, E)$ are disjoint $\gamma$-soft semiopen sets whose union is $\widetilde{X}$. This is the contradiction to our assumption. Hence $\widetilde{X}$ contains no nonempty proper subset which is both $\gamma$-soft semiopen and $\gamma$-soft semiclosed. 
(3) $\Rightarrow(1)$ : Suppose that $\widetilde{X}$ contains no nonempty soft subset which is $\gamma$-soft semiopen and $\gamma$-soft semiclosed and $\widetilde{X}$ is not $\gamma$-soft semiconnected. Then $\widetilde{X}$ can be expressed as the union of two nonempty disjoint soft sets $(A, E)$ and $(B, E)$ such that $\left((A, E) \widetilde{\cap} \tau_{\gamma^{-}} \operatorname{Cl}((B, E))\right) \widetilde{\cup}\left(\tau_{\gamma^{-}} \operatorname{Cl}((A, E)) \widetilde{\cap}(B, E)\right)=\widetilde{\emptyset}$. Since $(A, E) \widetilde{\cap}(B, E)=\widetilde{\emptyset},(A, E)=\widetilde{X} \backslash(B, E)$ and $(B, E)=\widetilde{X} \backslash(A, E)$. Since $\tau_{\gamma^{-}} \operatorname{Cl}((A, E)) \widetilde{\cap}(B, E)=\widetilde{\emptyset}, \tau_{\gamma^{-}} \operatorname{Cl}((A, E)) \widetilde{C} \widetilde{X} \backslash(B, E)$. Hence $\tau_{\gamma^{-}} \mathrm{Cl}((A, E)) \widetilde{C}(A, E)$. Therefore, $(A, E)$ is $\gamma$-soft semiclosed. Similarly, $(B, E)$ is $\gamma$-soft semiclosed. Since $(A, E)=\widetilde{X} \backslash(B, E),(A, E)$ is $\gamma$-soft semiopen. Therefore, there exists a nonempty set $(A, E)$ which is both $\gamma$-soft semiopen and $\gamma$-soft semiclosed. This is a contradiction to our assumption. Therefore, $\widetilde{X}$ is $\gamma$-soft semiconnected.

Theorem 4.10. An operation-soft topological space is $\gamma$-soft semiconnected if, and only if $\widetilde{X}$ is not the union of any two $\gamma$-soft semiseparated sets.

Proof. Let $(A, E)$ and $(B, E)$ be any two $\gamma$-soft semiseparated sets such that $\widetilde{X}=(A, E) \widetilde{\cup}(B, E)$. Therefore $\tau_{\gamma^{-s}} \mathrm{Cl}((A, E))$ $\widetilde{\cap}(B, E)=(A, E) \widetilde{\cap} \tau_{\gamma^{-}} \operatorname{Cl}((B, E))=\widetilde{\emptyset}$. Since $(A, E) \widetilde{\subset} \tau_{\gamma^{-}}$ $s \mathrm{Cl}((A, E))$ and $(B, E) \widetilde{\subset} \tau_{\gamma^{-} s} \operatorname{Cl}((B, E)),(A, E) \widetilde{\cap}(B, E)=\widetilde{\emptyset}$. Now $\tau_{\gamma^{-}} \operatorname{Cl}((A, E)) \widetilde{C} \widetilde{X} \backslash(B, E)=(A, E)$. So $\tau_{\gamma^{-}} \operatorname{Cl}((A, E))=$ $(A, E)$. Then $(A, E)$ is $\gamma$-soft semiclosed. By the same way we can show that $(B, E)$ is $\gamma$-soft semiclosed which contradicts with Theorem 4.9 (2). Conversely, let $(A, E)$ and $(B, E)$ be any two disjoint nonempty and $\gamma$-soft semiclosed sets over $X$ such that $\widetilde{X}=(A, E) \widetilde{\cup}(B, E)$. Then $\tau_{\gamma-s} \operatorname{Cl}((A, E)) \widetilde{\cap}(B, E)=$ $(A, E) \widetilde{\cap} \tau_{\gamma^{-} s} \mathrm{Cl}((B, E))=(A, E) \widetilde{\cap}(B, E)=\widetilde{\emptyset}$, which contradicts with the hypothesis.

Theorem 4.11. An operation-soft topological space is $\gamma$-soft semiconnected if, and only if for every pair of soft points $x_{\alpha}, y_{\beta}$ in $X$, there is a $\gamma$-soft semiconnected subset of $X$ which contains both $x_{\alpha}$ and $y_{\beta}$.

Proof. The necessity is immediate since the $\gamma$-soft semiconnected space itself contains these two points. For the sufficiency, suppose that for any two soft points $x_{\alpha}$ and $y_{\beta}$, there is a $\gamma$-soft semiconnected subset $(C, E)_{x_{\alpha}, y_{\beta}}$ of $\widetilde{X}$ such that $x_{\alpha}, y_{\beta} \in(C, E)_{x_{\alpha}, y_{\beta}}$. Let $a_{\mu}$ be a fixed soft point and $\left\{C_{a_{\mu}, x_{\alpha}}\right.$ : $\left.x_{\alpha} \widetilde{\in} \widetilde{X}\right\}$ be a class of all $\gamma$-soft semiconnected subsets of $\widetilde{X}$ which contain the points $a_{\mu}$ and $x_{\alpha}$. Then $\widetilde{X}=\underset{x_{\alpha} \widetilde{E} \widetilde{X}}{\cup} C_{a_{\mu}, x_{\alpha}}$ and $\underset{x_{\alpha} \in X}{\cap} C_{a_{\mu}, x_{\alpha}} \neq \widetilde{\emptyset}$. Therefore by Corollary 4.8, $\widetilde{X}$ is $\gamma$-soft semiconnected.

Theorem 4.12. Let $(X, \tau, E, \gamma)$ be an operation-soft topological space and $\left\{\left(F_{i}, E\right): i \in \Delta\right\}$ a family of $\gamma$-soft semiconnected sets. If a pair $\left(\left(F_{i}, E\right),\left(F_{j}, E\right)\right)$ is not a $\gamma$-soft semiseparation for any $i, j \in \Delta$, then $\widetilde{\cup}\left\{\left(F_{i}, E\right): i \in \Delta\right\}$ is $\gamma$-soft semiconnected.

Proof. Suppose $\widetilde{\cup}\left\{\left(F_{i}, E\right): i \in \Delta\right\}$ is not $\gamma$-soft semiconnected. Then there exist $\gamma$-soft semiseparated sets $(A, E),(B, E)$ such that $\widetilde{\cup}\left\{\left(F_{i}, E\right): i \in \Delta\right\}=(A, E) \widetilde{\cup}(B, E)$. Since $\left(F_{i}, E\right)$ is $\gamma$ soft semiconnected for each $i \in \Delta$ and $\left(F_{i}, E\right) \widetilde{\subset}(A, E) \widetilde{\cup}(B, E)$, by Theorem 4.3, $\left(F_{i}, E\right) \widetilde{\subset}(A, E)$ or $\left(F_{i}, E\right) \widetilde{\subset}(B, E)$. Now, put $\Delta_{a}=\left\{i \in \Delta:\left(F_{i}, E\right) \widetilde{\subset}(A, E)\right\}, \Delta_{b}=\left\{i \in \Delta:\left(F_{i}, E\right) \widetilde{\subset}(B, E)\right\}$. Then $\Delta_{a} \neq \widetilde{\emptyset}, \Delta_{b} \neq \widetilde{\emptyset}$ and $\Delta_{a} \widetilde{\cup} \Delta_{b}=\Delta$. Let $i_{a} \in \Delta_{a}$ and $i_{b} \in \Delta_{b}$, then $\left(F_{i_{a}}, E\right) \widetilde{\subset}(A, E)$ and $\left(F_{i_{b}}, E\right) \widetilde{\subset}(B, E)$. By Proposition 3.4, we obtain $\left(F_{i_{a}}, E\right)$ and $\left(F_{i_{b}}, E\right)$ are $\gamma$-soft semiseparated sets. This is contrary to our hypothesis.

\section{References}

[1] Abdulkadir Aygunoglu, Halis Aygun: Some notes on soft topological spaces. Neural Comput and Applic. DOI: 10.1007/s00521-011-0722-3.

[2] J. Biswas and A.R. Prasannan, An introduction to weaker and stronger form of soft open sets by $\gamma$-operation, Inter. J. Pure and Appl. Math., 116 (2) 2017, 285-298.

[3] Bozena Kostek: Soft set approach to the subjective assessment of sound quality. in: IEEE Conferences. 1 (1998), 669-674.

[4] D. Chen, E.C.C. Tsang, D.S. Yeung: Some notes on the parameterization reduction of soft sets, in: International Conference on Machine Learning and Cybernetics, vol. 3, 2003,pp. 1442-1445.

[5] D. Chen, E.C.C. Tsang, D.S. Yeung, X. Wang: The parameterization reduction of soft sets and its applications. Computers and Mathematics with Applications. 49 (2005), 757-763.

[6] Feng Feng, Young Bae Jun, Xianzhong Zhao: soft semirings. Computers and Mathematics with Applications. 56 (2008), 2621-2628.

[7] P. Gomathi sundari and B. Jaya Bharathi, Soft semiopen sets via operations (submitted).

[8] P. Gomathi sundari and B. Jaya Bharathi, Some weak forms of soft open sets via operations (submitted).

[9] Z. Kong, L. Gao, L. Wang, S. Li: The normal parameter reduction of soft sets and its algorithm, Computers and Mathematics with Applications 56 (2008), 3029-3037.

${ }^{[10]}$ E. F. Lashin, A. M. Kozae, A. A. Abo Khadra and T. Medhat: Rough set for topological spaces, Internat. J. Approx. Reason. 40 (2005), 35-43.

[11] D. Molodtsov: Soft set theory-first results. Computers and Mathematics with Applications. 37 (1999), 19-31.

[12] P.K. Maji, R. Biswas, A.R. Roy: Soft set theorys. Computers and Mathematics with Applications. 45 (2003), 555-562.

[13] P.K. Maji, A.R. Roy: An application of soft sets in a decision making problem. Computers and Mathematics with Applications. 44 (2002), 1077-1083.

[14] Milind M. Mushrif, S. Sengupta, A.K. Ray: Texture Classification Using a Novel, Soft Set Theory Based Classification Algorithm, Springer, Berlin, Heidelberg. (2006), 246-254.

[15] D. Pei, D. Miao: From soft sets to information systems, in: X. Hu, Q. Liu, A. Skowron, T.Y. Lin, R.R. Yager, B. 
Zhang (Eds.), Proceedings of Granular Computing, vol. 2, IEEE, 2005, pp. 617-621.

[16] S. Hussain and B. Ahmad: Some properties of soft topological spaces. Computers and Mathematics with Applications. 62 (2011), 4058-4067.

[17] M. Shabir, M. Naz: On soft topological spaces.Computers and Mathematics with Applications. 61 (2011), 1786-1799.

[18] Z. Xiao, L. Chen, B. Zhong, S. Ye: Recognition for soft information based on the theory of soft sets, in: J. Chen (Ed.), Proceedings of ICSSSM-05, vol. 2, IEEE, 2005, pp. 1104-1106.

[19] I. Zorlutuna, M. Akdag, W. K. Min, S. Atmaca: Remarks on soft topological spaces. To appear in Annals of Fuzzy Mathematics and Informatics.
$\operatorname{ISSN}(\mathrm{P}): 2319-3786$

Malaya Journal of Matematik

ISSN(O):2321 - 5666

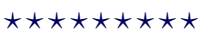

